\title{
Liquid noble gases for direct dark matter searches
}

\author{
Teresa Marrodán Undagoitia* \\ Max-Planck-Institut für Kernphysik, Saupfercheckweg 1, 69117 Heidelberg \\ E-mail: marrodan@mpi-hd.mpg.de
}

\begin{abstract}
Detectors using liquid noble-gas media like xenon or argon have shown a great potential for direct dark matter searches. Particles interacting with these targets cause the emission of light and free electrons, via excitation and ionization processes, respectively. The pulse shape of the prompt light signal and/or the ratio between light and extracted charge can be used to discriminate between different types of particles. For instance, nuclear recoils from dark matter interactions can be separated from electronic recoils from natural radioactivity, the main background contribution for this kind of experiments. In this paper, the different working principles of liquid noble-gas detectors are summarized. Furthermore, an overview of past, current and future experiments using the liquid noble gas technology is given, including the most important physics results and prospectives.
\end{abstract}

Technology and Instrumentation in Particle Physics 2014,

2-6 June, 2014

Amsterdam, the Netherlands

${ }^{*}$ Speaker. 


\section{Introduction to dark matter searches}

Cosmological and astronomical observations indicate the existence of a new form of nonluminous matter. These indications arise from very different phenomena and scales, from large scale structure-formation [1] and cosmic microwave background [2] to gravitational lensing [3] and rotation curves of stars in galaxies [4]. It is common to assume that this matter is made out of elementary particles. Such particles appear also as natural candidates in various models beyond the standard model of particle physics [5]. Specifically, the weakly interacting massive particle (WIMP) is a favoured candidate. WIMPs would have been in equilibrium in the early Universe and would have decoupled when the Universe expanded and cooled down [6].

If dark matter is made out of particles, there are three main possibilities to verify this: to produce them at particle accelerators, to look for products of their self-annihilations at locations where dark matter clusters, or to directly detect these particles via scattering off a detector's target material. A measurement of the latter type would provide information on the dark-matter particle mass and its interaction probability with ordinary matter.

Most direct detection experiments combine two types of signals (out of ionization charge, scintillation light or phonons in crystals) to discriminate nuclear recoils from electronic recoils. With this method, the main background which is mainly due to electronic recoils can be separated from possible signals due to dark matter collisions which would create nuclear recoils (see section 3). Other possible signal signatures are the annual modulation of the interaction rate resulting from the rotation of the Earth around the Sun (and through the WIMP wind) [7], the direction of the WIMPs [8] or the exponential shape of the spectrum at low nuclear recoil energies [9].

Indications for signals have appeared in few experiments, however, mostly at low statistical significance. An exception is the result of the DAMA experiment using NaI scintillating crystals, which has meanwhile a $9.3 \sigma$ evidence for an annual modulation of the scintillation rate. The phase of the modulation coincides with the expectation of being maximal around the 2nd of June [10]. If this signal is interpreted as being due to elastic scattering of dark matter (spin independent interaction), cross sections in the order of $2 \times 10^{-40}$ or $2 \times 10^{-41} \mathrm{~cm}^{2}$ at WIMP masses around 12 or $80 \mathrm{GeV} / \mathrm{c}^{2}$ are derived for scattering off $\mathrm{Na}$ or I, respectively [11] (see figure 2). Results from other experiments, however, are in strong tension with these results. At low WIMP masses, the germanium-based SuperCDMS [12] sets most constraining limit with cross sections down to $3 \times 10^{-42} \mathrm{~cm}^{2}$ at $7 \mathrm{GeV} / \mathrm{c}^{2}$ WIMP mass. For larger WIMP masses, liquid xenon-based experiments like XENON100 [13] and LUX [14] have set most stringent constrains down to $7.6 \times 10^{-46} \mathrm{~cm}^{2}$ at $33 \mathrm{GeV} / \mathrm{c}^{2}$ WIMP mass. During the last years, a large experimental effort has been made to determine and understand the energy scale and threshold in liquid xenon which is of relevance for the comparison with the results mentioned above (see section 4).

Astrophysical uncertainties on the velocity distribution of dark matter particles in the Milky Way halo can affect the interpretation of experimental results. It has been shown, however, that these uncertainties are not enough to solve the tension between the DAMA signal indication and the limits from other experiments [15]. In order to overcome this problem, an alternative representation of the data which integrates out astrophysical uncertainties has been proposed [16]. 


\section{Advantages of liquid noble gases}

Results from liquid noble gas detectors have shown the best sensitivities to WIMP interactions above $\sim 6 \mathrm{GeV} / c^{2}$ during the last years. Currently, mostly liquid argon (LAr) and liquid xenon (LXe) detectors exist or are under construction. However, there are some R\&D activities ongoing for liquid neon [17] as well. These media offer the possibility to achieve large and homogeneous targets with high scintillation and ionization yields. LAr and LXe scintillate both in the ultraviolet regime at $128 \mathrm{~nm}$ and $175 \mathrm{~nm}$, respectively [18]. For LAr, it is common to use wavelength shifters and detect light in the blue wavelength region while for LXe photosensors with quartz windows are used which are transparent to the xenon scintillation light.

Liquid noble-gas detectors offer two mechanisms to distinguish the main background due to $\gamma$ and $e^{-}$interactions (electronic recoils, ER) from the interactions of WIMPs with nuclei (nuclear recoils, NR). The scintillation pulse shape depends on the particle type because the so-called singlet and triplet states are populated at different ratios for ER and NR. This technique gives large discrimination power in liquid argon and liquid neon due to the very different lifetimes ( $6 \mathrm{~ns}$ and $1.6 \mu \mathrm{s}$ in LAr) [46] of the two components. In liquid xenon, the values for the decay constants are close to each other, $2 \mathrm{~ns}$ and about $30 \mathrm{~ns}$ [19], giving less rejection power. In addition to this method, the charge signal from ionization of the medium can be extracted and the ratio of charge to light can be used. Liquid xenon has also the advantage of containing almost $50 \%$ of non zero spin isotopes, ${ }^{129} \mathrm{Xe}$ and ${ }^{131} \mathrm{Xe}$, providing sensitivity to spin-dependent WIMP interactions [20].

Two detector concepts are currently being used: single-phase (liquid) and double phase time projection chambers (TPCs). The main features of each technology and the experiments which use them are described in sections 5 and 6.

\section{Background sources and reduction methods}

In order to identify unambiguously interactions from dark matter particles, ultra-low background experimental conditions are required. The background sources can be classified into nuclear recoil background from neutrons and electronic recoil background produced by $\gamma$ and $\beta$ decays. The nuclear recoil background arises from neutrons produced in $(\alpha, n)$ - and fission reactions from natural radioactivity, or due to spallation reactions of muons on nuclei in the experimental setup. This is a dangerous background because its signature is identical to the one of the WIMPs. Dark matter experiments are therefore, typically placed at underground laboratories in order to avoid the muon-induced neutrons. In addition, active or passive shields are built around the detector to reduce the neutron flux further. Furthermore, the detector medium and materials in the vicinity of the target need to have low concentrations of radioactive isotopes.

The electronic recoil background can be classified as external or internal. The external component originates from $\gamma$-decays in the materials surrounding the target. For large detectors, this background can be easily reduced by choosing an innermost volume for the analysis (fiducial volume). This is very effective in liquid xenon as its density of $\sim 2.8 \mathrm{~g} / \mathrm{cm}^{3}$ gives a strong self-shielding. Nevertheless, construction materials are screened and selected for lowest radioactive contaminations [21][22]. 
The internal background is distributed homogeneously in the medium and originates from radioactive isotopes of the target nuclei. For argon, ${ }^{39} \mathrm{Ar}$ with an endpoint energy at $565 \mathrm{keV}$ has a large contribution as it is produced from cosmic-ray activation at a level of $1 \mathrm{~Bq} / \mathrm{kg}$ in natural argon. In order to reduce it, argon from underground sources is extracted. It has been shown that in this way, the activity is reduced by at least a factor of 100 [23]. In xenon there is a double beta decaying isotope, ${ }^{136} \mathrm{Xe}$, however its lifetime is so large, $2.2 \times 10^{21} \mathrm{y}$ [24], that it doesn't contribute to the background for detectors up to few tons mass. In addition, backgrounds from ${ }^{85} \mathrm{Kr}$ and radon emanation from the detector materials contribute to the internal background. Krypton can be removed either by cryogenic distillation [25] or using chromatography [26]. Besides the reduction of krypton, methods to determine the remaining krypton contamination are being developed [27]. Recently, detections in the ppq (parts per quadrillion) regime of natural $\mathrm{Kr}$ in $\mathrm{Xe}$ have been achieved [28]. To reduce radon, a first approach is to use materials with low radon emanation. Furthermore, methods to remove the emanated radon continuously are being developed [29][30].

\section{Calibration}

For each liquid noble gas detector, the signal region (nuclear recoils) and the background region (electronic recoils) need to be characterised. Up to now, external gamma sources as ${ }^{137} \mathrm{Cs}$ or ${ }^{60} \mathrm{Co}$ have been used to determine the region in discrimination parameter where ER appear. However, as the detectors get larger (diameter of meters) the probability for single scatters of gammas in the centre decreases rapidly. The same argument applies for neutrons, however, due to their larger penetration length, external sources or generators can be used. In addition, the energy scale for both electronic and nuclear recoils needs to be determined. As the quenching processes (losses of signal) depend on the particle energy and its track characteristics [31], the energy scales are non linear and vary with applied drift field.

\subsection{Electronic recoil calibration}

In order to calibrate spatially uniform the electronic recoil band, new ideas involving internal sources have been proposed. The LUX experiment, for instance, has performed this calibration using tritiated methane [14]. Tritium undergoes beta decay resulting in a continuous electron spectrum with energies up to $18.6 \mathrm{keV}$.

For some dark matter candidates, the signature would consist of an electronic recoil. An example is the axion-like dark matter particle, see [32][33]. In this cases, the electronic recoil energy scale is needed and therefore, its linearity needs to be tested. A first approach was to use ${ }^{83 m} \mathrm{Kr}$ which is a decay product of ${ }^{83} \mathrm{Rb}$. This source has been tested in LXe both in single [34] and double phase [35] detectors and also in LAr and $\mathrm{LNe}$ [36] and provides calibration lines at $9.4 \mathrm{keV}$ and $31.2 \mathrm{keV}$. The low energy region relevant for dark matter searches is, however, at $\mathscr{O}(\mathrm{keV})$ or tens of $\mathrm{keV}$. Therefore, dedicated Compton experiments using a strong gamma source have been performed. By placing a coincidence detector at a certain angle, the kinematics are fixed and the energy deposition can be reconstructed. Results [38][39] show that there is a significant emission of light at energies as low as $1.5 \mathrm{keV}$. The light quenching at a drift field of $0.45 \mathrm{kV} / \mathrm{cm}$ is at the level of 75\% at these energies. The few measurements performed in liquid argon [36] show a constant behaviour of the ER light yield in the energy region from $40 \mathrm{keV}$ to $662 \mathrm{keV}$ at zero drift field. In 
general, when a field is applied, the overall light yield decreases because the scintillation light from electron recombination is reduced.

In order to describe scintillation and ionization processes at different energies, drift fields and media (LAr and LXe), a simulation framework has been developed. This model, called NEST [37], uses all existing measurements and describes the microscopic processes taking place during the production of photons and ionization electrons.

\subsection{Nuclear recoil calibration}

The signal region in dark matter detectors is determined using neutron sources which produce a nuclear recoil spectrum up to tens of keVs. In order to convert a measured signal in photoelectrons to $\mathrm{keV}$ recoil energies, the energy scale needs to be measured in addition. This scale is usually determined in dedicated laboratory setups by performing neutron scattering experiments. By setting a certain scattering angle, the corresponding nuclear recoil energy can be calculated from the kinematics. The light yield is related to the one of an electronic recoil such that the measurement is independent of the detector in use. The $122 \mathrm{keV}$ line of ${ }^{57} \mathrm{Co}$ is widely used for this purpose. The energy dependence of the nuclear recoils is then included in a function called $L_{e f f}$. This function can be seen for xenon in the left part of figure 1 and on the right for argon.
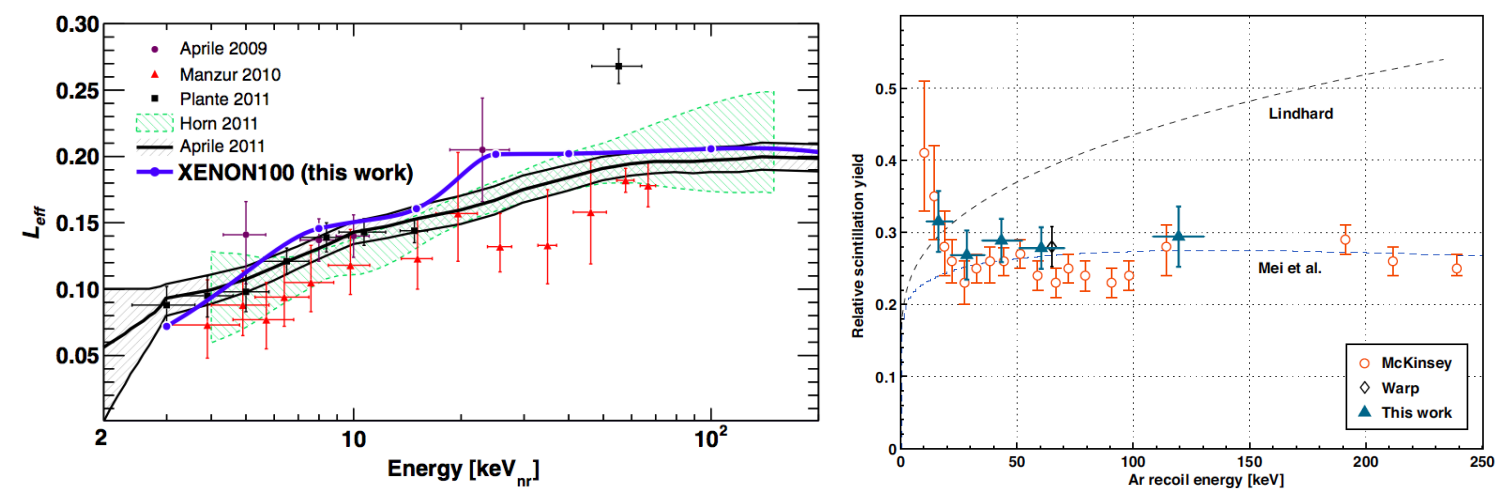

Figure 1: Energy dependence of the nuclear recoil quenching. The figure on the left shows $L_{e f f}$ for xenon from [42] and the figure on the right the relative quenching for argon from [43].

For xenon, a large set of measurements from neutron scattering experiments exist (see [40] for example), however, only the ones with smallest error bars are displayed in figure 1. Besides those, the results of Monte Carlo/Data comparisons of neutrons calibration sources from Zeplin [41] and XENON100 [42] are also included. Overall the measurements are in agreement with each other when the corresponding error bars are considered.

For argon, only few measurements exist. For energies above $30 \mathrm{keV}$ a flat behaviour is observed [43]. Below this energy the data points show increasing relative scintillation yields but the error bars are also larger. Commonly, energy thresholds of $(30-40) \mathrm{keV}$ are achieved in dark matter experiments using argon, and thus, the energy scale below these energies is not required. Recently, measurements of the drift field dependence of the scintillation output in LAr have been performed. It has been found that for $10 \mathrm{keV}$ nuclear recoil, a reduction of $30 \%$ in scintillation efficiency appears when applying $1 \mathrm{kV} / \mathrm{cm}$. The reduction is smaller for higher energies, around 
$15 \%$ for $50 \mathrm{keV}$ also at $1 \mathrm{kV} / \mathrm{cm}$ [44]. No indication for such field dependence is found in the existing data [45], however, this measurements are affected by large error bars and, therefore, new measurements are desirable.

\section{Single-phase detector technology}

Single phase detectors consist of a spherical target made out of liquid xenon or argon surrounded by photo-detectors. They have the advantage that a $4 \pi$-photosensor coverage can be realised giving a larger light output than detectors which are only partially instrumented. The distribution and timing of the photons at the photosensors can be used to determine the position of the event with $\mathrm{cm}$ resolution. The pulse shape is used to discriminate different types of particles. The pulses consist of two decay components, a fast (singlet) and a slow (triplet) one and the relative amplitudes depend on the particle type (see section 2).

The DEAP [47] and the CLEAN [48] are examples of LAr experiments which use this technology and are currently being constructed at the SNO laboratory in Canada. These detectors use light guides from the medium to the photosensor sphere in order to reduce the background from the radioactivity of the sensors. The DEAP-I prototype showed a discrimination power of $10^{-8}$ and an acceptance of $50 \%$ for nuclear recoils above an energy of $25 \mathrm{keV}$ [49]. As mentioned in section 2, this mechanism also works for liquid xenon [50] but not as efficient as in the argon case due to the similar decay components of the singlet and triplet states. The XMASS experiment [51] in Japan employs the single phase technology with liquid xenon. To further reduce the background, the detector is constructed using ultra-low radioactive materials. Self-shielding results in a radio clean innermost volume which is then used for analysis. In the latest run, an unexpected radioactive contamination of the photosensors appeared. Still some results on low WIMP masses [52] and on inelastic scattering off xenon [53] have been derived. Meanwhile the detector has been refurbished to shield the contamination from the PMTs and new data is expected soon. XMASS plans to extend to a 20 ton multi-purpose detector in the future [54].

\section{Double-phase detector technology}

Double phase detectors (liquid and gas) feature the possibility to detect both the scintillation light and the charge signal from ionization produced after an energy deposition [55]. The light is detected as prompt signal typically with two arrays of photosensors. The ionised electrons are drifted upwards to the liquid surface and amplified via proportional scintillation in the gas phase [56]. Double phase detectors are thus operated as a Time Projection Chamber (TPC) and are able to reduce the background using the charge/light ratio as this parameter depends on the particle type. In addition, fiducilization can be used as the position of the events can be reconstructed with $\mathrm{mm}$ resolution [57]. Event position reconstruction is performed by using the light pattern in the photosensors for the $(x, y)$-coordinates, and by obtaining the $z$ component from the time difference between the scintillation signal and the charge signal. TPCs have clear advantages for the detection for dark matter, however, it has to be considered that large detectors require to apply voltages of several tens of $\mathrm{kV}$ in order to achieve the drift fields needed for particle discrimination. 
In liquid argon detectors, the pulse shape features discussed in section 2 is used as an additional discrimination parameter. WARP [58] was the first LAr detector which delivered dark matter search results. It consisted of $2.3 \ell$ liquid argon and was located at the Laboratori Nazionali del Gran Sasso (LNGS) in Italy. Currently, a $50 \mathrm{~kg}$ detector, DarkSide [59], has been commissioned and is taking data at the same laboratory. A ton scale detector, ArDM [60] has been commissioned at CERN and has now been installed at the Canfranc underground laboratory in Spain.

From 2006 to 2011, the liquid xenon TPCs Zeplin [61] and XENON10 [62] showed the potential of this technology to measure dark matter. They placed most constraining exclusion limits on the coupling of dark matter particles to nuclei [63][64]. In 2009 XENON100 started operation at the LNGS laboratory. Its total mass of liquid xenon is $161 \mathrm{~kg}$, out of which $62 \mathrm{~kg}$ are contained inside the TPC. Careful screening and selection of radio-pure detector materials resulted in an electronic recoil background of $5.3 \times 10^{-3}$ events $\cdot \mathrm{kg}^{-1} \cdot \mathrm{d}^{-1} \cdot \mathrm{keV}^{-1}$ in the WIMP-search energy range for a $34 \mathrm{~kg}$ fiducial mass [65]. Currently, XENON100 continues to take data. However, in order to increase the sensitivity significantly, the XENON collaboration proposed a next generation detector, XENON1T [66], consisting of about 3 tons of LXe. The goal is to reduce the background by a factor of $\sim 100$ compared to the one of XENON100. This is achieved by using an additional water muon-veto detector, an improved material screening and selection and by reducing the intrinsic contamination with ${ }^{85} \mathrm{Kr}$ and radon using dedicated devices. In addition, a 3 inch high quantum efficiency and low radioactivity PMT (Hamamatsu R11410 [67]) will be used to further reduce the background. The experiment is built in such a way that an upgrade to XENONnT ( $\sim 6$ tons of LXe) can be performed with a moderate effort.

Figure 2 shows the experimental status as of July 2014. Closed regions represent signal indications while lines represent $90 \%$ confidence level exclusion limits on the WIMP-nucleon cross sec-

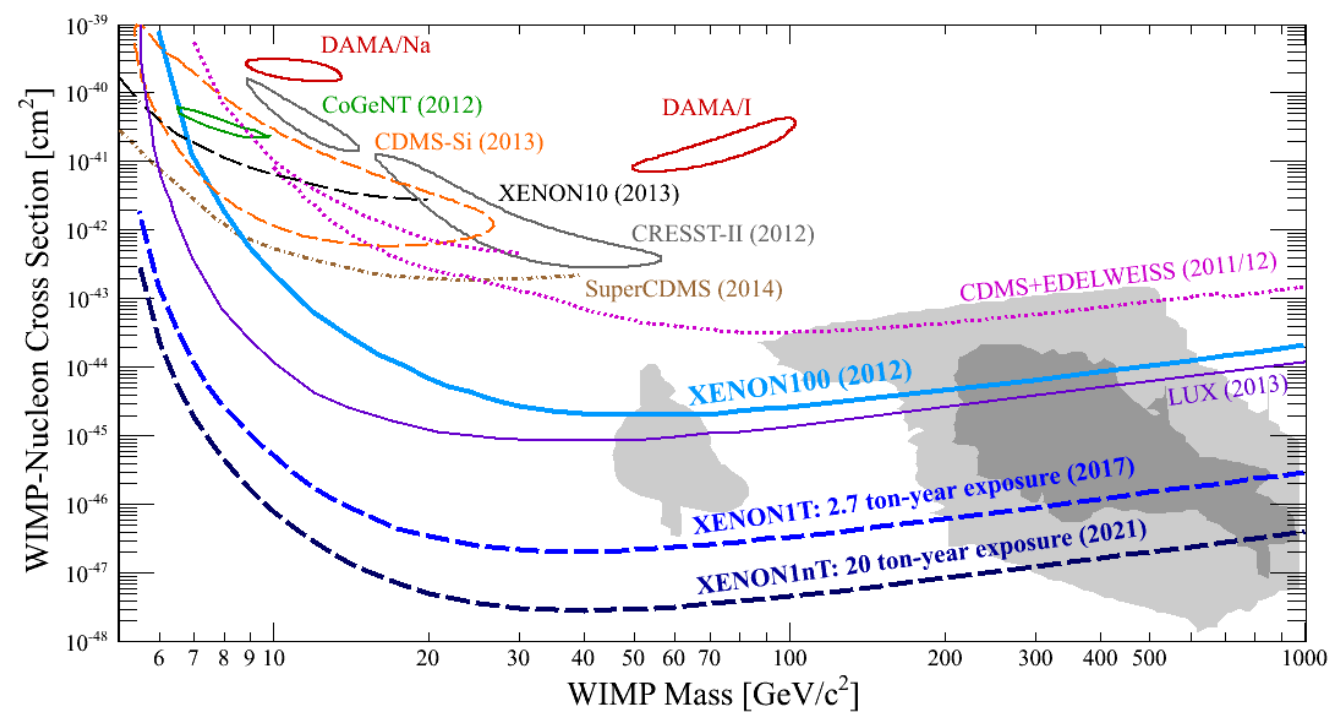

Figure 2: Exclusion limits and signal indications as of July 2014. Figure adapted from [13] including new results and the sensitivity projections for XENON1T and XENONnT. Note that projected sensitivities for other detectors (taking data, in construction or planned) are not displayed in this figure. 
tion. The grey shaded regions represent theoretically favoured parameters of the CMSSM, see [13] and references therein.

In 2013, the LUX experiment [26] which is installed at the Sanford underground laboratory in USA released first data [14] of 85 live days. These results confirm and improve the results of XENON100, in particular they are also in strong tension with signal indications from other experiments (see figure 2). Due to the larger light yield of the detector, 8 photoelectrons $/ \mathrm{keV}$ at $662 \mathrm{keV}$ energy, the experiment has set strong constrains at low WIMP masses. Plans for a multiton LXe detector, LZ [68], are currently on-going. Finally, a third LXe TPC, PandaX [69], has been constructed and commissioned in China and first results are expected soon.

The success of the liquid noble gas TPC technology has motivated further proposals for even larger detectors like the DARWIN (dark matter WIMP search with noble liquids) facility [70] in Europe, consisting of large liquid xenon and argon detectors. In the case of a dark matter signal evidence, DARWIN with a total mass of about 20 tons, could make a high statistics measurement of the dark matter particle properties, i.e. its mass and cross section. In addition, further physics channels including solar neutrinos, coherent scattering of neutrinos and neutrinoless double beta decay of ${ }^{136} \mathrm{Xe}$ could be measured in the liquid xenon part of DARWIN [71].

\section{Summary}

Liquid noble gases, especially detectors based on LXe TPCs, have shown a great potential to measure particle dark matter interactions. The self-shielding property and position reconstruction of the events make it possible to select the innermost volume of the detectors for analysis, resulting in very low background levels. Large detectors (up to ton-scale) are currently being constructed or planned. The advantages of this technology has resulted in world-leading sensitivities for crosssections of dark matter particles with ordinary matter.

\section{Acknowledgments}

Thanks to the conference organizers for the invitation to this interesting conference.

\section{References}

[1] V. Springel, C. S. Frenk and S. D. M. White, Nature 440 (2006) 1137 [astro-ph/0604561].

[2] G. Hinshaw et al. [WMAP Collaboration], Astrophys. J. Suppl. 208 (2013) 19 [arXiv:1212.5226].

[3] M. Bartelmann and P. Schneider, Phys. Rep. 340 (2001) 291, [astro-ph/9912508].

[4] V. C. Rubin, N. Thonnard, and W. Kent Ford, Astrophys. J., 225 (1978) L107.

[5] G. Jungman, M. Kamionkowski and K. Griest, Phys. Rept. 267 (1996) 195 [hep-ph/9506380].

[6] G. Gelmini and P. Gondolo, In G. Bertone (ed.): Particle dark matter 121 [arXiv:1009.3690].

[7] A. K. Drukier, K. Freese and D. N. Spergel, Phys. Rev. D 33 (1986) 3495.

[8] D. N. Spergel, Phys. Rev. D 37 (1988) 1353.

[9] J. D. Lewin and P. F. Smith, Astropart. Phys. 6 (1996) 87. 
[10] R. Bernabei et al., Eur. Phys. J. C 73 (2013) 2648 [arXiv:1308.5109].

[11] C. Savage, G. Gelmini, P. Gondolo and K. Freese, JCAP 0904 (2009) 010 [arXiv:0808.3607].

[12] R. Agnese et al. [SuperCDMS Collaboration], [arXiv:1402.7137].

[13] E. Aprile et al. [XENON100 Collaboration], Phys. Rev. Lett. 109 (2012) 181301 [arXiv:1207.5988].

[14] D. S. Akerib et al. [LUX Collaboration], Phys. Rev. Lett. 112 (2014) 091303 [arXiv:1310.8214].

[15] M. T. Frandsen et al., JCAP 1307 (2013) 023 [arXiv:1304.6066].

[16] P. J. Fox, J. Liu and N. Weiner, Phys. Rev. D 83 (2011) 103514 [arXiv:1011.1915].

[17] W. H. Lippincott et al. [MiniCLEAN Collaboration], Phys. Rev. C 86 (2012) 015807 [arXiv:1111.3260].

[18] O. Cheshnovsky, B. Raz and J. Jortner, Chem. Phys. Lett. 15 (1972) 475.

[19] A. Hitachi, T. Takahashi, N. Funayama, K. Masuda, J. Kikuchi and T. Doke, Phys. Rev. B 27 (1983) 5279.

[20] E. Aprile et al. [XENON100 Collaboration], Phys. Rev. Lett. 111 (2013) 2, 021301 [arXiv:1301.6620].

[21] G. Heusser, AIP Conf. Proc. 785 (2005) 39.

[22] L. Baudis et al., JINST 6 (2011) P08010 [arXiv:1103.2125].

[23] H. O. Back, F. Calaprice, C. Condon et al., [arXiv:1204.6024].

[24] J. B. Albert et al. [EXO-200 Collaboration], Phys. Rev. C 89 (2014) 015502 [arXiv:1306.6106].

[25] K. Abe et al., Astropart. Phys. 31 (2009) 290 [arXiv:0809.4413].

[26] D. S. Akerib et al. [LUX Collaboration], Nucl. Instrum. Meth. A 704 (2013) 111 [arXiv:1211.3788].

[27] E. Aprile, T. Yoon, A. Loose, L. W. Goetzke and T. Zelevinsky, Rev. Sci. Instrum. 84 (2013) 093105 [arXiv:1305.6510].

[28] S. Lindemann and H. Simgen, Eur. Phys. J. C 74 (2014) 2746 [arXiv:1308.4806].

[29] K. Martens et al. [XMASS Collaboration], Nucl. Phys. Proc. Suppl. 229 (2012) 562.

[30] S. Lindemann, PhD Thesis, University of Heidelberg (Germany) Nov. 2013

[31] M. Tanaka et al., Nucl. Instrum. Meth. A 457 (2001) 454.

[32] K. Abe et al. [XMASS Collaboration], Phys. Lett. B 724 (2013) 46 [arXiv:1212.6153].

[33] E. Aprile et al. [XENON100 Collaboration], [arXiv:1404.1455].

[34] L. W. Kastens, S. B. Cahn, A. Manzur and D. N. McKinsey, Phys. Rev. C 80 (2009) 045809 [arXiv:0905.1766].

[35] A. Manalaysay et al., Rev. Sci. Instrum. 81 (2010) 073303 [arXiv:0908.0616].

[36] W. H. Lippincott et al., Phys. Rev. C 81, (2010) 045803 [arXiv:0911.5453].

[37] M. Szydagis, A. Fyhrie, D. Thorngren and M. Tripathi, JINST 8 (2013) C10003 [arXiv:1307.6601].

[38] L. Baudis, H. Dujmovic, C. Geis, A. James, A. Kish, A. Manalaysay, T. Marrodán Undagoitia and M. Schumann, Phys. Rev. D 87 (2013) 115015 [arXiv:1303.6891].

[39] E. Aprile et al., Phys. Rev. D 86 (2012) 112004 [arXiv:1209.3658]. 
[40] G. Plante et al., Phys. Rev. C 84, 045805 (2011) [arXiv:1104.2587].

[41] M. Horn et al., Phys. Lett. B 705 (2011) 471 [arXiv:1106.0694].

[42] E. Aprile et al. [XENON100 Collaboration], Phys. Rev. D 88 (2013) 012006 [arXiv:1304.1427].

[43] C. Regenfus et al., J. Phys. Conf. Ser. 375 (2012) 012019 [arXiv:1203.0849].

[44] T. Alexander et al. [SCENE Collaboration], Phys. Rev. D 88 (2013) 9, 092006 [arXiv:1306.5675].

[45] A. Manzur, A. Curioni, L. Kastens, D. N. McKinsey, K. Ni and T. Wongjirad, Phys. Rev. C 81 (2010) 025808 [arXiv:0909.1063].

[46] M. G. Boulay and A. Hime, Astropart. Phys. 25 (2006) 179.

[47] M. G. Boulay [DEAP Collaboration], J. Phys. Conf. Ser. 375 (2012) 012027 [arXiv:1203.0604].

[48] K. Rielage et al. [MINICLEAN Collaboration], (2014) [arXiv:1403.4842].

[49] M. G. Boulay, B. Cai, M. Chen et al., (2009) [arXiv:0904.2930].

[50] K. Ueshima et al. [XMASS Collaboration], Nucl. Instrum. Meth. A 659 (2011) 161 [arXiv:1106.2209].

[51] K. Abe et al. [XMASS Collaboration], Nucl. Instrum. Meth. A 716 (2013) 78 [arXiv:1301.2815].

[52] K. Abe et al. [XMASS Collaboration], Phys. Lett. B 719 (2013) 78 [arXiv:1211.5404].

[53] H. Uchida et al. [XMASS Collaboration], PTEP 2014 (2014) 6, 063 C01 [arXiv:1401.4737].

[54] [XMASS Collaboration], http://www-sk.icrr.u-tokyo.ac.jp/xmass/index-e.html.

[55] A.I. Bolozdynya, Nucl. Instrum. Meth. A 422 (1999) 314.

[56] A. Lansiart et al., Nucl. Instrum. Meth. A 135, (1976) 47.

[57] E. Aprile et al. [XENON100 Collaboration], Astropart. Phys. 35 (2012) 573 [arXiv:1107.2155].

[58] R. Brunetti et al. [WARP Collaboration], Astropart. Phys. 28, (2008) 495.

[59] M. Bossa et al. [DarkSide Collaboration], JINST 9 (2014) C01034.

[60] A. Badertscher et al. [ArDM and CERN, PH/DT and Geneve Collaborations], [arXiv:1307.0117].

[61] D. Y. Akimov et al. [ZEPLIN Collaboration], Astropart. Phys. 27 (2007) 46 [astro-ph/0605500].

[62] E. Aprile et al. [XENON10 Collaboration], Astropart. Phys. 34 (2011) 679 [arXiv:1001.2834].

[63] D. Y. Akimov [ZEPLIN Collaboration] et al., Phys. Lett. B 709 (2012) 14 [arXiv:1110.4769].

[64] J. Angle et al. [XENON10 Collaboration], Phys. Rev. Lett. 100 (2008) 021303 [arXiv:0706.0039].

[65] E. Aprile et al. [ XENON100 Collaboration], Phys. Rev. D 83 (2011) 082001 [Erratum-ibid. D 85 (2012) 029904] [arXiv:1101.3866].

[66] E. Aprile et al. [XENON1T Collaboration], (2012) [arXiv:1206.6288].

[67] L. Baudis, A. Behrens, A. Ferella, A. Kish, T. Marrodán Undagoitia, D. Mayani and M. Schumann, JINST 8, P04026 (2013) [arXiv:1303.0226].

[68] D. C. Malling et al. [LZ Collaboration], (2011) [arXiv:1110.0103].

[69] X. Cao et al. [PandaX Collaboration], Sci. Chin. Phys. Mech. Astron. 57 (2014) 1476 [arXiv:1405.2882].

[70] L. Baudis et al. [DARWIN Consortium], PoS, IDM2010 122 (2010), [arXiv: 1012.4767].

[71] L. Baudis, A. Ferella, A. Kish, A. Manalaysay, T. Marrodán Undagoitia and M. Schumann, JCAP01 (2014) 044 [arXiv:1309.7024]. 